\title{
MACHIAVEL ET LA RHETORIQUE DES HUMEURS
}

\author{
Daniel Mansuy* \\ dmansuy@uandes.cl
}

RÉSUMÉ L'article ici présenté se propose d'analyser la nature des humeurs sociaux dans la pensée de Machiavel, à partir de l'interprétation de Claude Lefort. Le penseur florentin propose, dans Le Prince et les Discours, une théorie de la division sociale qui constitue une nouveauté dans l'histoire de la philosophie politique. Notre objectif est d'essayer de préciser la position de Machiavel face à ce conflit, et si celui-ci peut être surmonté.

Mots clés Machiavel, Claude Lefort, Philosophie Politique, Théorie Générale de l'État.

ABSTRACT Starting from an interpretation by Claude Lefort, the present article studies the nature of social humors in the thought of Machiavelli. In "The Prince" and in "The Discourses", the Florentine thinker proposes a theory of social division that is a novelty in the history of Political Philosophy. I try to determine Machiavelli's position vis-à-vis this conflict, and whether it can be overcome.

Keywords Machiavelli, Claude Lefort, Political Philosophy, General Theory of the State. 
Dans l'histoire des interprétations de Machiavel, l'ouvrage monumental de Claude Lefort, intitulé " Le travail de l'œuvre Machiavel », occupe une place privilégiée. En effet, il s'agit d'une exégèse fine et profonde qui, en outre, a creusé un sillon dont les effets sont loin d'être épuisés (Gaille, 2007 ; sur Lefort, voir Poltier, 1998 ; Manent, 2007a ; Labelle, 1998 ; Molina, 2005 ; Flynn, 2012 et Moreau de Bellaing, 2011). Un des grands mérites de Lefort est d'avoir centré sa lecture sur la question du conflit de classes. La lutte entre le peuple et les grands est, à ses yeux, centrale pour comprendre la portée de la pensée du Florentin, pensée difficile à saisir s'il en est (Poltier, 1998, p. 136). En outre, il est utile de rappeler que ce sujet n'a pas souvent été mis en avant par les grands courants d'interprétation. La lecture républicaine, par exemple, tend à ignorer ce problème. Et pour cause, dans la mesure où cette optique cherche à établir les points de continuité entre le républicanisme classique (grec ou romain) et Machiavel, elle a des difficultés à percevoir les éléments de rupture. Sur cette question, le Florentin rompt avec la tradition, au moins jusqu'à un certain point (Audier, 2005, pp. 30-31 et 271 ; Gaille, 2001, p. 237 ; McCormick, 2011, p. 8 ; Pocock, 1997 ; Skinner et coll., 1990). Ce problème demeure aussi relativement absent dans une autre grande interprétation de Machiavel, celle de Léo Strauss (1978), ce que Lefort ne manque pas de lui reprocher. Selon lui, Strauss s'est trop concentré sur le Machiavel religieux, négligeant le Machiavel politique qui analyse la société à partir de sa division (Lefort, 1986, pp. 302-304). D'après Lefort, Machiavel dresse une critique sévère de la compréhension classique du politique qui cherche à comprendre le phénomène politique à partir de la distinction et de la classification des régimes. Pour le Machiavel de Lefort, l'intelligence des questions politiques ne réside pas dans l'appréciation des formes de gouvernement, mais dans le jeu de forces sociales qui s'y organisent (Lefort, 1986, p. 474). Ainsi, par sa conception de la division sociale, le Florentin esquisserait une nouvelle ontologie fondée sur la division du désir humain (Manent, 2007a : 272-273). ${ }^{1}$

Dans les pages qui suivent nous nous intéresserons à la question du conflit de classes dans la pensée du Florentin, telle qu'elle est présentée dans « Le Prince » et les « Discours ». Notre objectif est d'essayer d'éclairer certains

1 En tout état de cause, nous ne voulons pas dire que la problématique du conflit ait été complètement ignorée avant "Le travail de l'œuvre Machiavel ». Qu'il suffise ici de nommer Sidney ou Montesquieu. En effet, Claude Lefort lui-même est l'héritier d'une certaine tradition qui a toujours mise en avant la division sociale. Une partie de cette tradition a été bien retracée dans l'ouvrage classique de James Burnham (1949). Raymond Aron, par exemple, s'inscrit dans cette lignée (voir Aron, 1993 et Audier, 2004). La vertu de Lefort est d'avoir fait une lecture extrêmement subtile et puissante des textes de Machiavel pour fonder sa propre perspective, et renouveler ainsi les études machiavéliennes. 
aspects de la théorie des humeurs, à partir des questions suivantes : s'il est vrai que les sociétés sont traversées par le conflit social entre deux groupes antagonistes, quels éléments définissent cette rivalité ? Quelle en est la profondeur? Autrement dit, nous chercherons à savoir en quoi réside la différence entre les deux humeurs, parce que si la compréhension du phénomène politique exige la connaissance des humeurs, il est alors indispensable d'en déterminer leur nature. Bien que notre point de départ soit l'interprétation de Claude Lefort, puisque ce dernier a ouvert une voie très féconde dans les études machiavéliennes, nous nous en éloignerons, notamment à propos du statut politique de la crainte, que Lefort tend à sous-estimer dans ses analyses. Notre hypothèse est que, pour rendre compte de la division sociale machiavélienne, il est indispensable de se référer à la peur, qui opère, sous certaines conditions, comme motif politique. Les hommes réagissant différemment face à la peur, la société tend à se diviser en deux groupes. Pour atteindre nos objectifs, nous analyserons dans un premier temps certaines observations relatives aux humeurs, présentes dans « Le Prince ». Puis, nous nous pencherons sur les propositions des « Discours », et leurs rapports avec la dynamique interne de la république romaine. Nous essaierons enfin de déterminer, à partir de la lecture des textes de Machiavel, le statut de la crainte dans la division sociale du Florentin. Nous tenterons, enfin, d'évaluer avec précision, la distance qui sépare Machiavel de Hobbes.

\section{Les humeurs au chapitre IX du Prince}

Au chapitre IX du « Prince », on trouve la première présentation explicite de la question des humeurs. Il s'agit d'un chapitre particulièrement complexe, où le seul titre — « Le principat civil » — pose des difficultés d'interprétation (Sasso, 1988 et Larivaille, 2001). Le principat civil, nous dit Machiavel, advient si une personne privée arrive au pouvoir non par la force mais avec le soutien des citoyens. Deux groupes peuvent le soutenir dans son accession au pouvoir : le peuple et les grands. Machiavel affirme qu'il existe ici une opposition radicale entre les deux groupes. Ceci exclut, par principe, toute solution de compromis entre eux. Autrement dit, un prince ne saurait accéder au pouvoir sans faire un choix entre le peuple et les grands : « En effet, dans toute cité, on trouve ces deux humeurs différents : et cela naît de ce que le peuple désire ne pas être commandé ni écrasé par les grands, et les grands désirent commander et écraser le peuple » (« Le prince », IX). ${ }^{2}$ 
Les appétits des deux groupes sont définis de façon parfaitement contradictoire : tandis que les grands veulent commander et écraser le peuple, celuici souhaite uniquement ne pas être opprimé. Arrêtons-nous sur ces éléments. D'un côté, notons que le désir du peuple est défini de façon purement négative : il ne s'agit pas, à proprement parler, d'un appétit mais d'un non-appétit, d'un non-désir. Claude Lefort parle d'une négativité pure, sans objet (Lefort, 1986, p. 477 ; voir aussi Lefort, 2007, p. 856). Si l'on en croit sa formulation, le peuple ne veut ni la concorde sociale ni le bonheur : le peuple est défini par la peur, sentiment entendu comme primaire et basique, presque comme un désir animal de sécurité. Nous verrons en tout cas que Machiavel va modifier par la suite sa position.

L'autre élément intéressant introduit par Machiavel est celui-ci: les deux appétits sociaux sont définis par une opposition antinomique, ce qui exclut a priori tout terrain d'entente. Il n'y a pas d'accord possible entre les deux groupes, pas de bien partagé à rechercher parce qu'il n'y a pas de véritable communauté politique. Celle-ci est scindée et semble irréconciliable. Machiavel s'éloigne ainsi de la philosophie politique classique en laissant supposer que toute recherche du bien commun n'est au fond qu'une manière plus ou moins déguisée de satisfaire l'un des deux appétits, généralement celui des grands. Donc, soit la philosophie politique est un leurre, car elle cherche quelque chose qui n'existe pas; soit elle se risque à justifier des appétits qui sont, par définition, injustifiables puisqu'ils vont au-delà de la justice : il n'y a pas, à proprement parler, de position impartiale. La politique se rapproche ainsi de la guerre, et par conséquent toute coopération devient suspecte (Luchese, 2010, p. 54). En même temps, il est nécessaire de remarquer que Machiavel écarte toutes considérations liées aux caractéristiques personnelles des membres de chaque groupe. Les habitudes ne sauraient jouer un rôle décisif, puisque c'est la position que chacun occupe dans l'espace social qui détermine ses appétits. Autrement dit, la nature de nos désirs est dictée par notre statut social. Cette idée, nous le savons, sera reprise et prolongée, par Karl Marx, à sa manière, dans sa «Critique de l'économie politique » (Marx, 1963, p. 273³).

3 «Dans la production sociale de leur existence, les hommes nouent des rapports déterminés, nécessaires, indépendants de leur volonté; ces rapports de production correspondent à un degré donné de développement de leurs forces productives matérielles. L'ensemble de ces rapports forme la structure économique de la société [...] Le mode de production de la vie matérielle domine en général le développement de la vie sociale, politique et intellectuelle. Ce n'est pas la conscience des hommes qui détermine leur existence, c'est au contraire leur existence qui détermine leur conscience ». Comparer aussi avec les observations faites par Aristote dans sa «Rhétorique »I, 10, 1369a6ss et II, 16, 1390b32ss. 
L'apport de Machiavel réside dans le fait qu'il dépasse la perspective classique, par les termes à l'aide desquelles il pose le problème. En effet, l'optique traditionnelle se révèle impuissante face à cette nouvelle définition du problème. Il s'agit d'un conflit qui ne saurait être résolu avec les outils de la philosophie classique parce que, à proprement parler, il est insoluble ${ }^{4}$ : il n'y a pas de mesure commune permettant d'évaluer les revendications des partis. C'est un problème qui, comme le notait encore Claude Lefort, nous confronte à la précarité et à la contingence de la réalité politique. L'existence même de la cité nous pose un problème sans solution, et l'art du politique — c'est un des enseignements du « Prince »- consiste justement à savoir apporter la réponse la plus adaptée aux circonstances. Néanmoins, le problème ne saurait se résumer à ces données abstraites : il est impossible à théoriser. L'usage même du terme humeurs, umori, suppose un enracinement profond dans la nature humaine, voire même une certaine animalisation de nos appétits (Fischer, 1997 et Manent, 2007a, p. 280) : si les intérêts peuvent, au moins en principe, trouver des points de convergence, il n'en va pas de même pour les humeurs, qui semblent irréductibles. ${ }^{5}$ On peut négocier sur des intérêts, mais pas sur des humeurs. Le politique doit s'atteler alors à une lourde tâche, celle de composer de façon la plus intelligente possible avec l'antinomie, puisqu'il n'y a pas, il ne peut y avoir un quelconque repos de la cité (Ménissier, 2010, p. 108).

Quoi qu'il en soit, Machiavel ne se contente pas d'énoncer les termes du problème. Le prince est certes confronté au conflit social, mais d'une façon toute particulière. En effet, celui-ci doit choisir sur quelle humeur prendre appui pour asseoir son pouvoir. Comme les inclinations de chaque groupe sont strictement contradictoires, le prince ne peut pas ménager les deux à la fois. Pour le Florentin, le choix le plus sage est celui du peuple, et il propose plusieurs arguments pour le prouver. D'abord, un prince ne peut ni commander ni manier à sa façon les gens qui « lui paraissent être ses égaux». Par contre, celui qui arrive au pouvoir avec la faveur populaire « s'y trouve seul », et n'a autour de lui personne " qui ne soit prêt à obéir ». Dans les mots de Claude Lefort, si le prince choisit de prendre appui sur ceux qui commandent, " il s'exposerait à voir son autorité sans cesse empêchée » (Lefort, 1986, p. 383). De surcroît, on ne saurait satisfaire les grands sans injustices. Au contraire, les appétits du peuple son plus honnêtes que ceux des grands. Machiavel introduit

4 C'est pourquoi Machiavel, malgré les premières pages des « Discours ", ne considère pas sérieusement le régime mixte, puisque, au fond, celui-ci recherche une certaine harmonie entre les partis en opposition (voir Luchese, 2010, pp. 110-111).

5 Pour une interprétation différente de la question des humeurs, voir Parel, 1992. 
ici un critère moral pour donner sa faveur à l'humeur populaire : ne pas vouloir être écrasé semble forcément plus honnête que de vouloir opprimer les autres. Mais, à la rigueur, ce n'est pas l'aspect moral des choses qui intéresse Machiavel, mais ses conséquences politiques (Lefort, 1986, p. 386). En effet, le Florentin ajoute encore un motif : le prince ne peut pas s'assurer du peuple, parce qu'il est très nombreux, mais il peut toujours $s$ 'assurer des grands, car il s'agit d'un groupe réduit par définition : la stratégie sert à faire " économie de violences » (Lefort, 1986, p. 386). Machiavel utilise ici un euphémisme assez récurrent dans « Le Prince » : en langage machiavélien, s'assurer veut dire tout simplement effacer du chemin. En outre, notre auteur affirme que d'un peuple ennemi on peut craindre l'abandon, mais les grands peuvent non seulement abandonner le prince, mais aussi se dresser contre lui. Enfin, le prince est obligé de vivre toujours avec le même peuple, mais pas avec les mêmes grands.

Pour illustrer l'importance d'avoir le peuple pour ami, Machiavel nous propose un exemple tiré de l'histoire : Nabis, tyran de Sparte, soutint un siège contre Grecs et Romains grâce à sa maîtrise de la division sociale. Il lui suffit, nous dit Machiavel, " de s'assurer de peu de gens ». En effet, ayant eu le peuple pour ennemi, il aurait été perdu. Machiavel veut prouver que le soutien du peuple est indispensable dans l'adversité, en tout cas plus important que celui des grands. Mais il y a un mot qui attire encore notre attention : Nabis s'est assuré des grands. Machiavel ne détaille pas davantage son propos, mais l'indication nous sert de piste. Nabis, raconte Tite-Live (qui semble être la source utilisé par Machiavel), utilisait la peur et les punitions sévères pour éviter les dissensions. Face aux périls venus de l'extérieur, il a dû prévenir toute trahison interne. Il a réuni les lacédémoniens pour leur expliquer que, face aux menaces externes, il était obligé de mettre en place des mesures de sécurité. Il annonça que certains citoyens seraient mis sous surveillance, mais il s'engagea à les libérer une fois les dangers dissipés. Quatre-vingt citoyens furent donc emprisonnés mais... exécutés la nuit suivante. Il fit fouetter ensuite des paysans suspects dans plusieurs villages, pour les tuer après. La terreur générée par ces actes paralysa les esprits et empêcha toute tentative de révolte (Tite-Live, 2001, pp. XXIV, 27). Les méthodes de Nabis sont donc, la ruse et la fraude pour éliminer tous les suspects, avant même qu'ils n'aient eu l'idée de se révolter. ${ }^{6}$ Ceci corrobore la propre affirmation de Machiavel, selon laquelle le prince doit craindre les grands comme s'ils étaient des ennemis déclarés (« 
Le prince » : IX). L'audace du fondateur, dans les mots de Lefort, « consiste justement en ceci qu'il bouleverse la hiérarchie établie, ruine le pouvoir des Grands et, pour édifier le sien, se tourne vers le peuple » (Lefort, 1986, p. 388), mais il le fait avec des moyens extrêmement violents, qui lui permettent de fixer, dans la mesure du possible, un nouvel ordre (Lefort, 1986, p. 494). Una fois le nouvel ordre intériorisé, le nouveau prince peut compter sur le peuple pour le soutenir (Lefort, 1986, p. 391). Il n'en reste pas moins que la position du prince est toujours instable, parce que fondée sur un déchirement social qui ne saurait être complètement comblé : aux yeux de Lefort, il y a là une critique implicite de Machiavel à l'ontologie aristotélicienne de la stabilité. Machiavel proposerait une nouvelle ontologie de la situation que rejette la philosophie aristotélicienne du repos et de l'ordre au profit d'une nouvelle considération des accidents, de l'histoire et, en définitive, du désordre (Lefort, 1986, pp. 357-358, 365-366 et 425 ; Manent, 2007a, pp. 272-273). ${ }^{7}$ C'est pourquoi il faut tenter de satisfaire et de canaliser les humeurs dans la mesure du possible. Cependant, l'équilibre sera toujours précaire et instable : c'est la condition du politique d'être toujours rattaché à une situation singulière. ${ }^{8}$ Autrement dit, la société machiavélienne est ouverte à l'évènement, ouverte au conflit insoluble entre les classes sociales (Lefort, 1986, p. 427). ${ }^{9}$

Dans tous les cas, le peuple paralysé de Nabis nous rappelle Borgia et Sévère, deux exemples de prince nouveau proposés par Machiavel. Borgia, par sa cruauté et son audace, laissa les peuples « satisfaits et stupéfiés » (« Le prince » : VII), tandis que les peuples de Sévère étaient « hébétés et stupéfaits » (« Le prince »: XIX). Cela peut éclairer notre question, parce que le conseil que Machiavel adresse au prince, celui, de prendre parti pour le peuple, ne tient pas à une quelconque raison morale ou démocratique. Tout simplement le peuple est plus facilement maniable que les grands, et donc le prince

7 Bien sûr, la critique implicite de la philosophie d'Aristote a une part d'injustice : le philosophe de Stagire est certainement l'un des penseurs qui a le plus soigneusement distingué le savoir théorique du savoir pratique, pour bien rendre la singularité de ce dernier. Mais, comme au livre XV du «Prince », Machiavel ne s'embarrasse pas des subtilités (sur ce problème, voir Manent, 2007a, pp. 269-271).

8 Ceci explique la critique extrêmement sévère que Machiavel adresse aux historiens qui l'ont précédé dans la Préface de ses « Histoires florentines »: en passant sous silence les problèmes liés aux discordes civiles, les historiens manquent l'essentiel et sont par-là incapables de rendre compte de l'histoire de Florence : « si quelque leçon est utile aux citoyens qui gouvernent les républiques, c'est la connaissance de l'origine des haines et des divisions », nous dit-il (« Histoires florentines », Préface, 945). Ce n'est pas la seule curiosité qui guide ici Machiavel : ceci touche à quelque chose d'essentiel de sa pensée politique. Pour lui, on ne peut pas expliquer l'histoire d'une cité, de ses grandeurs et ses misères, sans rendre compte des divisions qui l'ont agitée par le simple fait que ces conflits opèrent comme force motrice de l'histoire politique : les conflits sont la situation capable de rendre compte de toute réalité politique.

9 En même temps, le Florentin rompt avec Cicéron et l'héritage de l'humanisme classique, en récusant la distinction traditionnelle entre la paraison et la passion, l'homme et la bête (Lefort, 1986, pp. 410 et 771 ). 
parvient mieux à atteindre ses objectifs (Lefort, 1986, p. 424). Autrement dit, les grands peuvent toujours représenter un obstacle pour le prince : par leur pouvoir, par leur statut et par leur influence, ils ont toujours la possibilité de contrer ses projets. Si le prince veut asseoir sa position avec fermeté, il n'a pas vraiment le choix : il est obligé de les écarter de son chemin. Ceci a une double fonction : d'un côté, le prince ne rencontre plus de limites pour affermir son pouvoir et, d'un autre, la cruauté des exécutions paralyse le peuple et empêcha ainsi sa rébellion. De cette façon, toute révolte est tuée dans l'œuf, car les suspects sont éliminés, et le peuple terrorisé. On peut dire alors que le fait de s'assurer des grands permet, en même temps, de s'assurer du peuple. Le prince, et notamment le prince nouveau, peut ensuite donner la forme que bon lui semble à cette matière aussi souple que malléable, parce qu'elle n'a plus de réaction face au pouvoir (« Le prince »: VI). Le prince doit écraser toute ambition, en s'assurant des grands, et en laissant le peuple dans la stupéfaction, pour devenir enfin le maître incontestable de la situation : de cette façon, le maître n'a à dépendre que de soi (voir Lefort, 1986, p. 372).

\section{Les humeurs dans les « Discours»}

Tout au long des « Discours », Machiavel va revenir sur la question des humeurs. Bien entendu, ce texte ne met pas l'accent sur la figure du prince, mais les nombreuses variations introduites par Machiavel sont très utiles pour mieux comprendre la nature des partis, et le caractère du conflit. En même temps, il faut bien rappeler que les « Discours » opèrent un changement de ton par rapport au « Prince » : ce qui, dans l'opuscule, est dit de manière concise et brève, fait ici l'objet d'une attention plus détaillée, bien que peu systématique. Ceci vaut aussi pour le problème qui nous occupe puisque, dans les « Discours », Machiavel apporte constamment de nouvelles considérations qui éclairent progressivement son propos sur les humeurs, propos dont il est malaisé de faire la synthèse (Lefort, 1986, p. 460).

Dès les premiers chapitres de l'ouvrage, Machiavel fait référence au problème de la division sociale. On sait que l'histoire des divisions romaines a été longuement décrite par Tite-Live. Cependant, si ces luttes sont considérées par l'historien comme un phénomène plutôt négatif, Machiavel y voit une clé lui permettant de comprendre l'histoire et la grandeur romaine. Il sait bien que la perspective qu'il propose suppose une innovation majeure - qui d'ailleurs lui sera reprochée par Guichardin et Harrington (Guichardin, 2000, p. 344 ; Harrington, 1995, pp. 237, 259)—, et il prévient alors son lecteur : mon regard sur 1'histoire de Rome, nous dit-il, sera différent des opinions reçues (voir par 
exemple Augustin, 2001 : II, 18-19 et III, 16-17). Selon ces dernières, Rome doit sa grandeur à la fortune et à la vertu militaire, et les tumultes étaient normalement rangés parmi les défauts de Rome. Machiavel prend le contre-pied de cette opinion courante : "Je dis que ceux qui condamnent les tumultes entre les nobles et la plèbe me semblent blâmer ce qui fut la cause première du maintien de la liberté de Rome et accorder plus d'importance aux rumeurs et aux cris que ces tumultes faisaient naître qu'aux bons effets qu'ils engendraient » (« Discours »: I, 4).

Les auteurs précédents sont aveugles, dit-il, puisqu'incapables d'apprécier les effets positifs des tumultes et des divisions : ils restent dans le domaine de l'apparence, et victimes d'une illusion. Ils manquent ainsi l'élément central, puisque, à ses yeux, la division civique produit la liberté : « Il y a dans chaque état deux humeurs différentes, celle du peuple et celle des grands, et [...] toutes les lois que l'on fait en faveur de la liberté naissent de leur désunion » (« Discours »: I, 4). ${ }^{10}$

La dichotomie, comme dans « Le Prince », se veut universelle : il y a, dans tous les états, deux humeurs différentes. Un peu plus tard, il revient sur cette idée : « Celui qui considère les choses présentes et les anciennes comprend facilement que dans toutes les cités et dans tous les peuples il y a les mêmes désirs et les mêmes humeurs, et qu'ils y ont toujours été » («Discours »: I, 39).

Machiavel revient ainsi à une formulation des humeurs concordante avec celle du Prince, dans le contexte de ses réflexions sur Rome. En tout cas, il n'y a pas de place pour le doute : la division sociale n'est pas une singularité romaine, mais une réalité consubstantielle à la politique et aux sociétés humaines. La singularité romaine réside dans le fait d'avoir su maîtriser cette division, et qui sait maîtriser ce conflit détient la clé du savoir politique (Lefort, 1986, p. 476).

La longue comparaison entreprise par Machiavel, dès les premiers chapitres des « Discours », entre le modèle romain d'un côté et le modèle conservateur de Venise et Sparte de l'autre, peut nous aider à cerner sa pensée. Nous savons qu'elle conclut en faveur de Rome, parce que cette dernière a su profiter du dynamisme issu de la logique conflictuelle. Venise et Sparte on fait un choix conservateur, caractérisé par le calme et la stabilité. Rome, pour sa part, a suivi un chemin différent, qui consiste à assumer la dynamique politique en se servant de la force générée par la division (Lefort, 1986, p. 480). Machiavel son « Discours sur la réforme de l'État à Florence fait sur la demande de Léon X » (1955, Vol. II, p. 431). 
admet volontiers qu'il y ait de bonnes raisons pour préférer la voie conservatrice, mais les choses politiques possèdent une dynamique qui leur est propre, et en conséquence, dit-il, " la nécessité vous entraîne à beaucoup des choses auxquelles la raison ne vous entraîne pas » (« Discours » : I, 6). Autrement dit, le désordre peut produire un ordre extrêmement puissant (Lefort, 1986, p. 470).

Le schéma conservateur, qui attribue tout le pouvoir à une classe dominante, n'a de sens que si la cité ne cherche pas à conquérir mais seulement à conserver. Cependant, ce désir s'avère, dans les faits, irréaliste, parce que tout désir de conservation se transforme, qu'on le veuille ou non, en désir d'acquisition. Les conservateurs ne peuvent l'être pendant très longtemps : ils sont obligés de modifier leur attitude à l'épreuve des faits (Flynn, 2012, p. 93). Ceci explique l'extraordinaire puissance du modèle romain : il est le mieux adapté à la réalité politique, et son dynamisme est le mieux adapté à la vérité effective de la chose (Lefort, 1986, p. 553). Les conflits sociaux sont, nous dit Machiavel, " un inconvénient nécessaire pour parvenir à la grandeur » («Discours » : I, 6), ce qui veut dire que la politique démocratique est, en même temps, une politique agressive vers l'extérieur (Lefort, 1986, p. 553). De façon pertinente, Pierre Manent a défini la pensée du Florentin comme une philosophie visant à accueillir le mouvement du monde : il ne s'agit pas de nier ou de neutraliser les conflits, mais de savoir les utiliser au profit de la cité (Manent, 2010, pp. 260-264 et Lefort, 1992, pp. 175-176). Autrement dit, la politique digne de ce nom est celle qui sait accueillir le mouvement du monde, au lieu de le nier (Molina, 2005, p. 182). La leçon de Machiavel semble donc être celle-ci: il faut savoir se servir des inévitables discordes civiles. Les conservateurs ont peur des divisions, et cette peur entraîne des conséquences fâcheuses. D'un côté, elle les prive des puissantes forces sociales que Rome a su mettre en œuvre jusqu'à conquérir le monde. D'un autre, cette peur conduit à des impasses politiques, parce qu'elle mène à une espèce d'aveuglement face à la division des classes.

\section{Vers une compréhension des humeurs}

Il est donc clair que la distinction entre les deux groupes joue un rôle tout à fait central dans la pensée machiavélienne. Mais, tout en avançant et développant sa compréhension des choses, Machiavel nous livre différentes caractérisations des humeurs, qui vont nuancer subtilement ses formulations les plus visibles sur la question. De manière générale, ses sympathies explicites vont au peuple, et nous avons vu que dans « Le Prince » 1'humeur populaire 
était même considérée comme étant «plus honnête » (« Le prince »: IX). Ceci explique pourquoi il contredit les historiens pour assurer la défense du peuple, tout en suggérant que ceux-ci prenaient la défense des grands pour des motifs peu avouables («Discours » : I, 58). Machiavel veut changer de camp, il veut être le premier démocrate de l'histoire : le premier philosophe à ne pas assumer le point de vue des Grands (Manent, 2007a, pp. 279-280). Mais en regardant les choses de près, il n'en reste pas là : sa position est plus complexe, puisqu'il modifie progressivement son premier enseignement sur la question des humeurs. Rappelons quelques passages qui pourraient nous aider à mieux comprendre ses propos.

Au chapitre $4 \mathrm{du}$ livre premier des « Discours », Machiavel affirme qu'il faut que le peuple puisse donner libre cours à ses « ambitions ». Les désirs populaires, poursuit notre auteur, sont rarement pernicieux pour la liberté puisqu'ils surgissent soit du fait d'être opprimés, soit de la peur de l'oppression («Discours » : I, 4). Machiavel indique ici, de manière indirecte mais, nous semble-t-il, assez claire, que la peur, sentiment purement négatif, est à l'origine d'un sentiment plutôt positif : l'ambition. Autrement dit, le désir de ne pas être opprimé n'est pas une pure négativité, comme le prétend par moments Claude Lefort (1986, p. 477); en tout cas, c'est une négativité qui peut se métamorphoser. En fait, la peur peut tout à fait engendrer des dispositions actives, elle est bien à l'origine d'un désir. Ainsi, la peur n'est pas qu'enfermement. La peur du peuple dérive vers une ambition qui lui est propre, et qui enclenche d'ailleurs une dynamique toute particulière, comme nous le verrons. D'un autre côté, les appétits des grands possèdent aussi leur complexité, et ne sont pas statiques : ceux qui veulent conserver veulent, en fait, acquérir davantage, la classe possédante n'est pas une classe qui cherche la stabilité (Manent, 2007a, p. 274). C'est pourquoi Machiavel reformule les termes de la question : les conservateurs sont davantage des ambitieux que des conservateurs. De leur côté, les désirs populaires subissent une poussée parallèle : ne pas vouloir être opprimé entraîne à vouloir acquérir ; le désir du peuple contient haine et envie, ce qui l'éloigne de la pure négation. Claude Lefort admet volontiers ce dernier point (Lefort, 1986, pp. 479, 483 et 485) mais il considère que cela n'empêche pas l'humeur populaire de rester toujours identique à elle-même.

Quelques pages plus tard, Machiavel approfondit ce point de vue, en semant encore le doute quant à la dimension négative de l'humeur populaire. En effet, au cours du chapitre 37, il essaye d'éclairer les causes de la décadence de Rome, en s'arrêtant longuement sur la discussion de la loi agraire, qui a été, à son avis, l'une des causes de la décadence romaine. Les hommes, nous dit le Florentin, s'affligent dans le mal et se lassent dans le bien; ils ne savent pas 
être en repos (« Discours »: I, 37). ${ }^{11}$ On retrouve alors un argument analogue à celui qu'il avait utilisé pour établir la supériorité de Rome face à Sparte et Venise : le repos est peut-être désirable, mais il n'est pas compatible avec notre nature (voir Lefort, 1986, p. 478). Appliqué à Rome, ce principe nous donne à voir une autre caractéristique de l'humeur populaire : en fait, la plèbe n'a jamais été satisfaite du pouvoir qu'elle obtenait par les conflits successifs, et cherchait toujours à en avoir davantage. Elle ne pouvait pas faire autrement : dans l'anthropologie qui est celle de Machiavel, les hommes sont condamnés à être insatisfaits. La plèbe ne s'est pas contentée des tribuns, puisqu'elle a voulu obtenir ensuite des honneurs et des biens (voir Sullivan, 2011, p. 177 et Najemy, 2010, p. 110). Le jugement de Machiavel est très sévère sur ce point : les plébéiens n'ont jamais trouvé entière satisfaction. Les raisons du succès romain permettent aussi de rendre compte de sa décadence : le conflit dégage des énergies permettant d'atteindre la grandeur, mais la logique conflictuelle ne saurait avoir de limites, ni obtenir complète satisfaction. Étant donné la précarité de l'équilibre politique, les conflits peuvent très bien être aussi la source de la ruine de la cité, lorsqu'ils excèdent un certain cadre. ${ }^{12} \mathrm{C}$ 'est exactement ce qui est arrivé à Rome lors de la discussion sur la loi agraire : la plèbe, en exigeant toujours davantage, a provoqué une tension très difficile à maîtriser qui a produit, à terme, la guerre civile et la chute de la république. Le désir de ne pas être opprimé est un désir qui n'est jamais satisfait, ni complètement innocent (Lefort, 1986, p. 520). En fait, le caractère oppressif de tout ordre social est indépassable, ce qui empêche le désir populaire de trouver le repos (Lefort, 1992, p. 173).

Nous sommes loin de la caractérisation purement négative de l'humeur populaire ; en tout cas, il y a un certain nombre d'objections à prendre en compte. Celle-ci a subi une sorte de métamorphose : si dans un premier temps le peuple ne ressent qu'une peur négative et sans objet, il développe progressivement un désir d'acquérir analogue à l'appétit des grands, même s'il reste soumis à une logique différente. Il est vrai que, au commencement, il y avait la peur ; mais une fois que la plèbe sort de son état primaire, sa crainte laisse place à d'autres inclinaisons plus actives (« Histoires florentines »:

11 II dit aussi que « la nature a créé les hommes de façon telle qu'ils peuvent tout désirer et ne peuvent pas tout obtenir ; si bien que, le désir étant toujours supérieur au pouvoir d'acquérir, il en résulte le mécontentement de ce qu'on possède, et le peu de satisfaction qu'on en a » (177). Voir aussi « Histoires florentines », III, 11, IV, 13 et V, 14 («l'ambition humaine n'a pas de bornes »). En tout cas, cette réalité était connue de la philosophie classique (voir «Politique », II, 7, 1267b3-4), seulement les Grecs y attribuaient une signification différente.

12 Cet aspect de la pensée machiavélienne comporte un caractère aristotélicien : le conflit suppose une communauté politique, faute de quoi il devient destructeur (Audier, 2005, p. 297). 
IV, 14). Cela peut contribuer à expliquer le singulier conseil machiavélien, selon lequel toute république, tous les dix ans, doit se servir de la terreur pour revenir aux commencements (« Discours »: III, 11). Dans un certain sens, revenir aux principes implique de revenir à cette peur dépourvue de tout désir d'acquérir, et cela ne peut pas se faire sans terreur, parce que c'est la seule façon de récupérer cette peur originelle indispensable à tout ordre politique (nous avons vu l'exemple de Nabis qui, dans une certaine mesure, vaut pour les républiques et les principats). Faute de retour aux commencements, la plèbe en voudra toujours plus, parce que son appétit est impossible à satisfaire complètement. La peur primitive peut se manifester avec timidité (voir Montesquieu, 1993, pp. I, 2 ; et Rousseau, 1959, p. 787) ; mais, une fois enclenchée la logique des appétits (ce qui est inévitable dans un contexte politique) on ne saurait l'arrêter sans avoir recours à la terreur. En tout cas, dans la mesure où la seule manière de tout donner à la plèbe est de tout enlever aux grands, on peut supposer que le destin inévitable de toute république qui a mise en marche cette dynamique est la tyrannie d'un seul : les « Discours » retrouvent ainsi la logique interne du « Prince ».

Mais revenons un instant au texte. Dans le chapitre 16, toujours au livre I, Machiavel se demande comment le prince doit traiter un peuple ennemi. Il s'agit d'un passage où le Florentin adopte un ton républicain, en même temps qu'il livre quelques remarques sur l'humeur populaire. On peut, nous dit-il, distinguer deux appétits au sein du peuple («Discours » : I, 16). D’une part le désir de vengeance à l'égard de ceux qui l'ont opprimé et, d'une autre, celui de récupérer la liberté. On savait déjà que l'humeur populaire pouvait générer l'ambition, mais nous savons désormais qu'elle peut aussi engendrer le désir de vengeance. Le peuple ne se satisfait ni de la fin de l'oppression, ni de l'acquisition du pouvoir : il veut se venger. Bien entendu, rien de plus facile pour un prince que de satisfaire cet appétit : ici, les intérêts du prince et du peuple sont parfaitement convergents, et les propos des Discours sont une nouvelle fois cohérents avec ceux du « Prince».

En tout état de cause, le peuple ne réclame pas seulement la vengeance, il veut aussi la liberté. Mais, comment caractériser ce désir de liberté ? Machiavel introduit ici une nouvelle distinction, fondamentale à nos yeux : pour certains, la liberté consiste à vivre en sécurité ; tandis que pour d'autres, la liberté implique le commandement. Le passage est particulièrement difficile, mais le Florentin semble faire une double suggestion. D'abord, il laisse entendre que, pour un grand nombre, la liberté se réduit au désir de sécurité : la liberté serait alors équivalente au désir de ne pas être écrasé, tout en sachant que ce désir ne peut pas être satisfait sans vengeance. Dans ce sens, la liberté 
politique exige une certaine garantie par rapport à l'oppression, tout en conservant un fondement dans la peur. Mais le texte va plus loin, en opérant une division au sein même du désir du peuple. Dans la plèbe, il y a ceux qui veulent vivre en paix et en sécurité, mais il y a également ceux qui veulent commander. Autrement dit, le système binaire de deux humeurs est reproduit à l'intérieur même du peuple : dans tout groupe humain, certains veulent commander, ils veulent la liberté pour dominer les autres (voir Coby, 1999, p. 94 et Lefort, 1986, p. 496). Cela implique que concéder du pouvoir au peuple ne saurait résoudre le conflit, parce que la division se recrée au sein même du peuple : la plèbe produit ses propres grands. Autrement dit, l'élimination de la classe dominante ne conduit pas à une société homogène, mais à une nouvelle division. C'est l'une des leçons, centrale pour l'intelligence du XXe siècle, que Claude Lefort a tirée de la lecture de Machiavel (Audier, 2005, pp. 236-237). Aucune société ne parvient à dépasser cette réalité : la division sociale est constitutive du monde humain. Dans tous les cas, si l'appétit de ceux qui veulent la sécurité est facile à satisfaire, gérer l'appétit de ceux qui veulent commander s'avère beaucoup plus difficile. Machiavel envisage deux options : soit $s$ 'assurer du groupe qui veut commander, soit lui offrir des honneurs. Mais le conflit est, évidemment, insoluble : si on élimine le groupe qui veut commander, le peuple aura tendance à reproduire les mêmes ambitions ; si on lui donne des honneurs, ses membres voudront en avoir davantage par la suite (voir aussi « Discours » : II, 23). C'est le cercle infernal de la politique, ou du moins de la politique machiavélienne, où les ambitions des hommes ne sauraient trouver un quelconque repos. Le conflit est inscrit dans la configuration de toute société humaine, et les humeurs ne sont que la manifestation politique de ce fait.

On voit bien que la première distinction, selon laquelle il y aurait deux humeurs complètement antinomiques, s'atténue considérablement. Il semble en effet que les humeurs soient moins contradictoires et moins divergentes que les premières formulations ne le laissaient entendre. Nous trouvons une confirmation de cette hypothèse dans le chapitre 5 du livre I. Dans ce passage, Machiavel entreprend la comparaison entre Rome et Sparte, à laquelle nous avons fait allusion, la question y étant de savoir s'il est plus sûr de donner la charge de la liberté aux grands ou au peuple. Machiavel critique le point de vue traditionnel selon lequel ceux qui possèdent sont mieux placés pour exercer des responsabilités politiques. Il est plus sûr, nous dit-il, de donner cette charge au peuple parce que le grand nombre a moins d'appétit d'usurpation que le petit nombre. Cette discussion lui permet de revenir sur la caractérisation des deux humeurs : les nobles ont un désir de domination, tandis que le 
peuple désire ne pas être dominé. Cette considération amène Machiavel à se poser la question suivante : quel appétit est le plus pernicieux pour une république ? La formulation de la question est intéressante en soi, parce qu'elle suppose que les deux appétits sont nocifs, la différence étant seulement dans le degré. Une interrogation vient alors à l'esprit du lecteur : comment un appétit purement négatif pourrait-il être nocif pour la liberté ? La suite nous apporte la réponse :

On débattit alors beaucoup pour savoir qui est le plus ambitieux —celui qui veut maintenir ou celui qui veut acquérir-, car l'un et l'autre de ces appétits peuvent facilement être à l'origine de très grands désordres. Néanmoins, la plupart du temps, ils sont causés par ceux qui possèdent, car la peur de perdre produit chez eux les mêmes envies que ceux qui désirent acquérir (« Discours » : I, 5).

Il nous semble que ce texte a une portée considérable. D'un côté, il opère un glissement : de purement négatif, l'appétit du peuple se transforme en désir d'acquérir. En ce sens, il contredit ouvertement ce que Machiavel avait dit un peu plus tôt, dans le même chapitre. En fait, le supposé non-désir du peuple se transforme en désir de posséder. Les deux appétits, qui avaient été si radicalement opposés, sont ici réduits au même phénomène, et presque inversés, parce que ce sont les grands qui ont maintenant une attitude plutôt défensive : à la limite, ils ne veulent que conserver. Mais la conclusion est que tous ont « les mêmes envies » (le medesime voglie) : les humeurs ont subi une transformation brutale. Il est certain que, à l'origine de chaque envie, on trouve des humeurs différentes mais, dans leur cristallisation effective, les humeurs tendent à se rapprocher, et Machiavel n'hésite pas à les mettre sur un pied d'égalité. ${ }^{13}$ Et comment pouvait-il en être autrement si l'on tient compte de l'anthropologie prêchée tout au long des « Discours »?

Les deux partis de la cité sont, au même titre, à l'origine des désordres civils. La suite du texte confirme notre lecture, parce que Machiavel indique que le comportement des grandes peut faire naître une nouvelle division dans le désir de la plèbe. Le peuple peut avoir soit une soif de vengeance, qui l'entraîne à dépouiller les nobles; soit une envie d'accéder aux mêmes honneurs et richesses : on voit bien que Machiavel se réfère toujours aux mêmes alternatives. En cela, le Florentin avance une affinité certaine entre les deux appétits : leur distinction n'est que situationnelle, ce qui implique que leur différence n'est pas radicale. En fait, c'est une nature humaine identique qui peut réagir ceux à qui le royaume revient, mais aussi dans le cœur de ceux à qui il ne revient pas » (401). 
différemment selon qu'elle se trouve d'un côté ou d'un autre de la cité, mais c'est la même nature qui est toujours à l'œuvre. Il y a donc une certaine proximité entre les deux appétits.

En ce sens, les interprétations qui mettent trop l'accent sur la radicalité de la distinction entre les deux appétits nous semblent insuffisantes. Gérald Sfez, par exemple, insiste sur la radicale hétérogénéité des humeurs. Pour lui, le désir du peuple est " minime et absolu », et en fait le peuple " insiste radicalement dans son refus du pouvoir ». Il s'agit donc de " deux désirs de nature différente ou hétérogène ", qui n'admettent pas de commune mesure. En fait, pour Sfez, la rivalité entre les deux humeurs ne s'établit pas autour d'un même enjeu, « mais dans la tentative d'imposer à l'autre l'enjeu qui est le sien », puisque chaque désir " prétend à l'universalité de son enjeu » (Sfez, 1995, pp. 17-18). McCormick ne dit pas autre chose. À ses yeux, les deux appétits sont « qualitativement différents (2011, p. 5). Serge Audier, quant à lui, pense qu'il s'agit de " deux désirs qui ne relèvent pas du même ordre » (2005, p. 213). Cette perspective se risque à perdre de vue un aspect essentiel du problème : s'il est vrai que, au commencement, les humeurs apparaissent sous un jour différent, ces différences s'atténuent une fois leur dynamique enclenchée. Il est évident qu'on ne peut pas nier la présence constante des deux humeurs dans l'œuvre machiavélienne. Seulement, à trop insister sur leur différence, on néglige un aspect central : les deux appétits sont produits par la même passion, la peur. Les grands veulent écraser les petits parce qu'ils ont peur de perdre ce qu'ils possèdent. De leur côté, les pauvres craignent à priori les riches ; mais, étant donné, que la vie sociale n'est pas un élément statique, les appétits commencent à se rapprocher. Comme l'affirme Patrick Coby, la description des humeurs proposée par Machiavel implique que, au fond, les humeurs tendent à la même chose (1999, p. 94 ; Flynn, 2012, p. 92). C'est la peur qui peut produire des effets différents selon les circonstances et l'endroit où l'on est placé : dans ce sens, il n'y a pas d'enjeux radicalement différents, mais un même sentiment (la crainte) qui peut avoir des traductions politiques différentes selon la position de l'agent politique. Autrement dit, à l'origine des humeurs, l'on trouve, comme le note Harvey Mansfield, des dispositions pré-rationnelles qui se manifestent de manière différentes, mais dont les enjeux ne sont pas radicalement éloignés (Mansfield, 1996, p. 24). Cependant, il n'en demeure pas moins que la société humaine tend à se diviser, constitutivement, en deux groupes. Pour preuve, la plèbe elle-même produit cette division. Les humeurs ne sont pas radicalement différentes, mais ce n'est pas pour autant que le problème politique est résolue ni que les appétits sont conciliés : Machiavel n'envisage pas, parce qu'il ne le croit pas possible, une 
politique où la peur et la division pourraient être neutralisées. ${ }^{14}$ En effet, la peur propre aux hommes engendre le désir d'acquérir richesses et honneurs, mais — comme d'ailleurs le note Sfez lui-même (1995, p. 13) - ces objets de désir sont de nature nécessairement comparative : on désire toujours avoir davantage que le voisin, et toute comparaison suppose un point commun. En effet, la peur et le désir qui l'accompagne sont consubstantiels à la nature de tous les hommes. Thierry Ménissier voit juste: toutes les actions, de tous les hommes, de toutes les classes, sont mues par cette pulsion; et c'est d'ailleurs dans cette conception que réside la rupture machiavélienne (Ménissier, 2010, pp. 145 et 153 ; Lefort, 1992, pp. 166-167). En tout cas, dans la mesure où Machiavel n'envisage aucune pacification des passions — ce qui sera la tâche de la philosophie politique à partir du XVII siècle (Hirschmann, 1997)_, il ne croit pas possible d'effacer le conflit de l'horizon humain : la peur, telle qu'elle est comprise par le Florentin, est intrinsèquement conflictuelle. C'est peut-être Hugues Poltier qui a le mieux réussi à rendre compte du problème, dans son effort d'appréhender la philosophie de Claude Lefort. À ses yeux, " le désir du sujet humain se brise dans deux pôles incompatibles », et " le désir de l'homme échoue nécessairement à s'accomplir, brisé qu'il est en deux exigences contraires et également impérieuses » (Poltier, 1998, pp. 145-146). Il y a bel et bien un désir humain, mais qui se brise inévitablement sitôt qu'il existe Cette explication a l'avantage de concilier l'unité du désir humain (que Machiavel affirme explicitement à plusieurs reprises) avec l'impossibilité de trouver une solution au conflit : ce n'est pas parce que c'est le même désir qu'on peut envisager pour autant une pacification du conflit : les concrétisations des désirs sont rigoureusement incompatibles (Lefort, 1986, p. 404).

Bien entendu, Claude Lefort sait bien que le désir du peuple se divise luimême, et que la peur populaire engendre des dispositions qui vont au-delà de la pure négativité. Mais, pour lui, il n'en reste pas moins qu'il y a toujours une partie du peuple, la plus grande d'ailleurs, qui ne développe aucun désir de pouvoir, qui subit toujours l'oppression (Lefort, 1986, pp. 384 et 521) ; dans ce sens, on ne saurait dire que les deux désirs soient équivalents (Lefort, 1986, p. 729). Autrement dit, s'il est vrai que le peuple se divise et engendre de luimême une classe dominante, cela vient seulement confirmer sa propre thèse : à l'origine, il y a toujours une division sociale. L'ordre humain ne saurait être

14 Dans les «Discours », il n'envisage même pas une classe moyenne qui pourrait tempérer les appétits, comme l'avait fait Aristote («Politique » IV, 11, 1295b2-3 ; V, 3 et 11 ; voir sur ce sujet Edmond, 2000, p. IX). En tout cas, dans les "Histoires florentines ", Machiavel considère une classe moyenne avec des formulations qui tendent à contredire les enseignements des « Discours " "Comme il y avait trois sortes de peuple, puissant, moyen et petit », II, 42). Voir aussi son « Discours sur les choses de Florence ». 
ramené à une quelconque unité, parce que la division lui est constitutive : tel serait le message machiavélien. Tout cela est parfaitement recevable, et cohérent avec le texte machiavélien, mais l'affirmation symétrique doit être énoncée en même temps : à l'origine, il y a la peur; et c'est cette deuxième assertion qui, par moments, est absente des analyses de Lefort. ${ }^{15} \mathrm{C}$ 'est la peur qui engendre deux sentiments, et en conséquence deux effets politiques d'ordre différent (et c'est ce dernier fait qui semble surtout intéresser Lefort, 1986, pp. 729, 733), mais cette division contient à l'origine une certaine unité. Bien sûr, Lefort connaît parfaitement l'importance de la peur comme motif politique dans la pensée de Machiavel, mais il met au première plan de la dynamique politique non pas la peur, qui serait seulement un stade premier, mais la nécessité découverte par le savoir politique, à partir de la division sociale, d'agir ici et maintenant pour libérer l'action : le philosophe ne serait plus impartial. À certains égards, dans l'interprétation de Lefort, le Machiavel politique l'emporte sur le Machiavel philosophe ou, plutôt, se confond avec lui (Lefort, 1986, pp. 529-530). Après tout, la grande vertu de l'humeur populaire est de permettre l'ouverture de l'espace public, et d'empêcher l'oppression perpétuelle (Lefort, 1986, pp. 485 et 522-524). Cependant, comme le note Pierre Manent, le risque de l'oppression, même démocratique, demeure toujours présent ; et c'est pourquoi ni la constatation de la division ni la faveur populaire ne sauraient être des critères ou des étalons pour évaluer les ordres politiques (Manent, 2007a, p. 283). La question qui s'impose, à laquelle nous ne pouvons pas répondre ici, est celle de savoir si les exigences de la politique sont compatibles avec celles de la philosophie ; autrement dit, si le Machiavel politique, qui veut libérer les espaces d'action et qui nous oblige à abandonner notre position de témoin pour rejoindre celle du peuple (Lefort, 1986, p. 476) ne pratique pas un certain art d'écrire à ce sujet. ${ }^{16}$ On peut penser que c'est l'une des grandes interrogations que l'œuvre de Lefort nous impose. ${ }^{17}$

Ces considérations peuvent nous guider sur la voie d'une autre grande tâche qui consiste à mesurer la distance exacte qui sépare Machiavel de

15 Notons, pour le reste, que Claude Lefort a modifié sa compréhension des humeurs. Comparer par exemple les articles suivants : «Machiavel et la veritá effetuale » et « Machiavel : la dimension économique du politique », in «Les formes de l'histoire : essais d'anthropologie politique », Gallimard, 1978, 127-140. Dans le premier article il nie que les humeurs machiavéliens puissent être réduites à une question économique, pour accepter cette hypothèse dans le second. L'auteur derrière cette hésitation est, bien sûr, Marx, pour qui le conflit est avant tout et fondamentalement économique, ce qui ne l'empêche pas de croire à son caractère irréductible.

16 Pour le problème de l'impartialité du philosophe, voir Manent, 2007a, p. 279.

17 Miguel Vatter radicalise la thèse de Lefort : à ses yeux, le philosophe français ne voit pas la tension entre liberté politique et forme politique, et ne perçoit pas que toute forme d'institution (même la règle de la loi) cache une certaine forme d'oppression (Vatter, 2014, pp. 16, 245, 295, passim). 
Hobbes. On sait que le fondement de l'anthropologie politique hobbesienne est la peur, jointe à « un désir perpétuel et sans trêve d'acquérir pouvoir après pouvoir » (Hobbes, 1999, p. XI), ce qui produit un état de guerre et de misère : aucun véritable progrès n'est possible dans un tel contexte. Il semble donc inévitable de voir une certaine parenté entre l'anthropologie de Hobbes et celle de Machiavel. Au fond, ils partagent une conception pessimiste de la nature humaine : ce qui commande notre nature, c'est le désir d'acquérir (Ménissier, 2010, p. 145). Mais nous avons insisté sur le fait que Machiavel ne croit pas à une quelconque pacification des désirs, il ne croit pas à une institution qui puisse neutraliser le désordre induit pas les passions humaines, tandis que tout l'effort de Hobbes tend à nous montrer comment les hommes pourraient élaborer un dispositif artificiel pour sortir du cercle de la violence. Machiavel ne le croit pas possible, Hobbes se différencie de lui : s'il conserve la thèse machiavélienne selon laquelle les hommes sont gouvernés par le désir d'acquérir, il ajoute une deuxième prémisse, qui bouleverse complètement le schéma du Florentin. En effet, pour Hobbes l'état de nature est un état de parfaite égalité : « la différence d'un homme à un autre, dit-il, n'est pas si considérable qu'un homme puisse de ce chef réclamer pour lui-même un avantage auquel un autre ne puisse prétendre aussi bien que lui » (Hobbes, 1999, p. XIII). Hobbes fonde son système sur cette hypothèse : à l'origine les hommes sont égaux. Pour Machiavel, nous l'avons suffisamment vu, les pulsions humaines se divisent, dès le premier moment, en deux pôles incompatibles, en deux tendances divergentes (Poltier, 1998, pp. 145-156). L'unité des désirs humains ne peut être ramenée à un ordre quelconque, il n'y a pas d'institution susceptible de réussir cet exploit : la peur ne fonde pas l'égalité mais, bien au contraire, elle engendre une division insurmontable. De son côté, Hobbes tente d'apprivoiser par le biais des institutions, l'animal machiavélien (Manent, 2007b, pp. 61-62). C'est pourquoi Machiavel veut nous laisser face à un problème qui n'admet pas de solution, tandis que Hobbes essaye de nous montrer la voie de sortie. Dans ce sens, affirmer la primauté de la peur chez Machiavel le rapproche certainement de Hobbes ; mais affirmer en même temps la division sociale, l'en tient à une distance non négligeable. En définitive, pour Machiavel, tout « Léviathan », tout effort pour neutraliser complètement la situation originale, reste illusoire, ce qui explique en partie sa position paradoxale dans l'histoire de la pensée politique : Machiavel est un moderne sceptique sur la possibilité d'apporter une quelconque solution au problème politique. Sur ce point, il reste un moderne tout à fait singulier. ${ }^{18}$ 


\section{Références}

ARISTOTE. « Les politiques ». Flammarion, 1999.

ARISTOTE. « Rhétorique ». Flammarion, 2007.

ARON, R. « Machiavel et les tyrannies modernes ». Fallois, 1993.

AUDIER, S. "Machiavel, héritier du républicanisme classique », Cahiers de philosophie de l'Université de Caen, 34, pp. 9-35, 1996.

. « Raymond Aron : la démocratie conflictuelle ». Michalon, collection « Le bien commun », 2004.

. « Machiavel, conflit et liberté ». Vrin-Ehess, 2005.

AUGUSTIN. « La cité de Dieu ». Gallimard, Pléiade, 2001.

BENNER, E. “Machiavelli's Prince. A New Reading”. Oxford University Press, 2013.

BURNHAM, J. « Les machiavéliens défenseurs de la liberté ». Calmann-Lévy, 1949.

COBY, P. "Machiavelli's Romans. Liberty and Greatness in the Discourses on Livy". Lexington Books, 1999.

EDMOND, M. P. « Aristote. La politique des citoyens et la contingence ». Payot, 2000.

FISCHER, M. « Machiavelli's Political Psychology ». The Review of Politics, 59, n. 4, pp. 789-829, 1997.

FLYNN, B. « La philosophie politique de Claude Lefort ». Belin, 2012.

GAILLE, M. « Machiavel au prisme du moment machiavélien ». pp. 231-240. In : G. Sfez, M. Senellart (eds.). L'enjeu Machiavel. Puf, Collection « Librairie du collège international de philosophie », 2001.

2007.

. " Machiavel et la tradition philosophique », Puf, collection « Philosophies »,

GUICHARDIN, F. « Condiderazioni intorno ai Discorsi del Machiavelli ». Einaudi, 2000.

HARRINGTON, J. « Océana ». Traduction de P. F. Henry, revue et complétée par François Delastre. Belin, collection « Littérature et politique », 1995.

HIRSCHMANN, A. O. «Les passions et les intérêts. Justifications politiques du capitalisme avant son apogée ». Puf, 1997.

HOBBES, T. «Léviathan. Traité de la matière, de la forme et du pouvoir de la république ecclésiastique et civile ». Dalloz, 1999.

LABELLE, G. « De l'œuvre de la pensée à la question du politique. La question de l'interprétation chez Claude Lefort ». In : L. Olivier et coll.. Épistémologie de la science politique. Presses Universitaires du Québec, 1998.

LARIVAILlE, P. « Chapitre IX du Prince : la crise de la principauté civile », pp. 81103. In: Y. Ch. Zarka, Th. Ménissier. Machiavel, le Prince ou le nouvel art politique. Puf, collection « Débats philosophiques », 2001.

LEFORT, C. « Machiavel : la dimension économique du politique », pp. 127-140. In : . Les formes de l'histoire : essais d'anthropologie politique. Gallimard, 1978. . « Le travail de l'œuvre Machiavel ». Gallimard, collection « Tel», 1986. 
. "Machiavel et la veritá effetuale », pp. 141-179. In : Écrire. À l'épreuve de la politique. Calmann Levy, 1992.

. «Pensée politique et histoire. Entretien avec Pierre Pachet, Claude Mouchard, Claude Habib et Pierre Manent ». In : . Le temps présent. Écrits 1945-2005. Belin, 2007.

LUCHESE, F. del. " Tumultes et indignation. Conflit, droit et multitude chez Machiavel et Spinoza ». Éditions Amsterdam, 2010.

MACHIAVEL. « Histoires florentines ». In : Euvres complètes. Gallimard, collection « Bibliothèque de la Pléiade », 1952.

. « Discours sur la réforme de l'État à Florence fait sur la demande de Léon $\overline{\mathrm{X} » . \text { In }}$ : Toutes les lettres officielles et familières (édition d'Edmond Barincou). Gallimard, 1955.

. "Le Prince ». Edition bilingue de Jean-Louis Fourmel et Jean-Claude Zancarini. Puf, collection "Fondements de la politique", 2000.

. " Discours sur la première Décade de Tite-Live ». Traduction d'Alessandro Fontana et Xavier Tabet, Gallimard, collection « Bibliothèque de philosophie », 2004. MANENT, P. « Vers l'œuvre et vers le monde. Le Machiavel de Claude Lefort ». In : . Enquête sur la démocratie. Gallimard, collection « Tel», 2007a.

. « Naissances de la politique moderne ». Gallimard, collection « Tel», 2007b. . «Les métamorphoses de la cité. Essai sur la dynamique de l'Occident ». Flammarion, 2010.

MANSFIELD, H. « Le prince apprivoisé. De l'ambivalence du pouvoir ». Fayard, collection «L'esprit de la cité, 1994.

. « Machiavelli’s Virtue ». University of Chicago Press, 1996.

$\overline{\text { MARX }}, \mathrm{K}$. « Critique de l'économie politique ». In : CEuvres, Économie I. Gallimard, collection «Bibliothèque de la Pléiade », 1963.

McCORMICK, J. P. « Machiavellian Democracy ». Cambridge University Press, 2011.

MÉNISSIER, T. « Machiavel ou la politique du centaure ». Hermann, 2010.

MOLINA, E. « Le défi du politique. Totalitarisme et démocratie chez Claude Lefort ». L'Harmattan, 2005.

MONTESQUIEU. « De l'esprit des lois ». Flammarion, 1993.

MOREAU DE BELLAING, L. «Claude Lefort et l'idée de société démocratique ». L'Harmattan, 2011.

NAJEMY, J. M. «Society, Class and State in Machiavelli's Discourses on Livy », pp. 96-111. In:

University Press, 2010. (éd.). The Cambridge Compation to Machiavelli. Cambridge PAREL, A. J. «The Machiavellian Cosmos ». Yale University Press, 1992.

POCOCK, J. G. A. « Le moment machiavélien : la pensée politique florentine et la tradition républicaine atlantique ». Puf, collection « Léviathan », 1997.

POLTIER, H. « Passion du politique. La pensée de Claude Lefort ». Labor et Fides, 1998. 
ROUSSEAU, J.-J. « Rousseau juge de Jean-Jacques ». In : Euvres complètes, Vol. 1. Gallimard, collection «Bibliothèque de la Pléiade », 1959.

SASSO, G. «Principato civile e tirannide ». In: Machiavelli e gli antichi, Vol. II. Milano-Napoli, Ricciardi, 1988.

SFEZ, G. « Machiavel : la raison des humeurs ». Rue Descartes, 12-13, pp. 11-37, 1995.

SKINNER, Q. et coll. (eds.). " Machiavelli and republicanism ». Cambridge University Press, 1990.

STRAUSS, L. « Thougths on Machiavelli ». Chicago University Press, 1978.

SULLIVAN, V. B. « Machiavelli’s Three Romes ». Northern Illinois University Press, 2011.

TITE-LIVE. « Histoire de Rome ». Flammarion, 2001.

VATTER, M. « Between Form and Event. Machiavelli's Theory of Political Freedom ». Fordham University Press, 2014. 